\section{OP16 IMPACT OF POVERTY AND FAMILY ADVERSITY ON ADOLESCENT HEALTH. A MULTI-TRAJECTORY ANALYSIS USING THE UK MILLENNIUM COHORT STUDY}

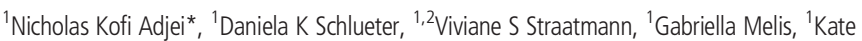
M Fleming, ${ }^{3}$ Ruth McGovern, ${ }^{4}$ Louise Howard, ${ }^{3}$ Eileen Kaner, ${ }^{5}$ Ingrid Wolfe, ${ }^{1}$ David C Taylor-Robinson. 'Department of Public Health, Policy and Systems, University of Liverpool, Liverpool, UK; ${ }^{2}$ Department of Public Health Sciences, Stockholm University, Stockholm, Sweden; ${ }^{3}$ Population Health Sciences Institute, Newcastle University, Newcastle, UK; ${ }^{4}$ Department of Health Service and Population Research, King's College London, London, UK; ${ }^{5}$ Department of Women and Children's Health, King's College London, London, UK

\subsection{6/jech-2021-SSMabstracts. 16}

Background Both poverty and family adversities including domestic violence, parental mental illness and parental drug and alcohol use are associated with poor outcomes across the life course. However, the complex relationships between these exposures in childhood are unclear. We therefore assessed the clustering of trajectories of household poverty and family adversities and their impacts on child health outcomes in adolescence.

Methods We used longitudinal data from the nationally representative UK Millennium Cohort study on 11564 children born between 2000 and 2002, followed through six survey waves. Family adversities were defined here as parent reported domestic violence and abuse, parental alcohol use and parental mental illness. We used a group-based multi-trajectory cluster model to define trajectories of poverty and family adversity for children aged 9 months to 14 years. We assessed associations of these trajectories and child outcomes at age 14 years (child socioemotional behavioural problems, cognitive disability, obesity, alcohol and drug use) using multivariable logistic regression adjusting for confounders.

Results Six trajectories were identified: persistent alcohol misuse $(7.7 \%)$, low poverty and family adversity (43.2\%), persistent domestic violence and abuse (3.4\%), persistent parental mental illness (11.9\%), persistent poverty $(22.6 \%)$ and poverty and parental mental illness (11.1\%). Compared to the low poverty and family adversity trajectory, children in the other trajectory groups experienced worse outcomes, particularly for combined exposure to poverty and parental mental illness. Compared with children with low adversity, those in the parental mental illness and poverty group were particularly at increased risk of socioemotional behavioural problems (adjusted OR 6.4, 95\% CI 5.0 - 8.3), cognitive disability (aOR 3.1, CI 2.4 - 4.2), drug use (aOR 1.7, CI 1.4 - 2.0) and obesity (aOR 1.8, CI $1.3-2.5$ ).

Conclusion Over half of children in the UK grow up experiencing poverty and adversities associated with poor health in adolescence. Persistent poverty and/or persistent parental mental illness affect over four in ten UK children. The combination of both affects one in ten and is very strongly associated with adverse child outcomes, particularly poor child mental health.

\section{OP17 WHICH INTERVENTIONS IMPROVE SLEEP DURATION IN CHILDREN? A SYSTEMATIC REVIEW AND META- ANALYSIS OF RANDOMIZED CONTROLLED TRIALS}

Lucia Magee*, Umar Chaudhry, Claire Nightingale, Angela Donin, Liz Stovold, Lucy Goldsmith, Charlotte Whalich, Alicja Rudnicka, Chris Owen. Population Health Research Unit, St. George's University London, UK

10.1136/jech-2021-SSMabstracts. 17
Background Adequate sleep duration in childhood has important implications for social, mental, and physical well-being. Recent evidence has demonstrated declining sleep durations in children; therefore effective interventions to improve sleep measures are needed. Despite some trials suggesting that multi-behavioural interventions promoting bed-time routines may be beneficial, the effectiveness of such interventions has not been adequately quantified. Moreover, specific themes and strategies which increase sleep duration, and by how much, have not been formally quantified.

Methods We carried out a systematic review and meta-analysis to quantify the effectiveness of sleep interventions to improve sleep duration in children within community settings (PROSPERO ID CRD42019160089). Studies involving infants under 1 year, those using medications, and targeting children with behavioural problems were excluded. We screened 11621 randomised control trials (RCT), after carrying out a search using combined text words and MeSH criteria in CENTRAL, MEDLINE, EMBASE, PSYCHINFO, Web of science, clinicaltrials.gov, and WHO trials databases. Full text screening of 341 studies identified 39 studies for data extraction. All screening was carried out independently by two reviewers using a web-based platform for systematic reviews.

Results Studies were categorised by age as follows: 20\% in $>1-5$ years of age, $25 \%>5-10$ years, $33 \%>10-15$ years, $22 \%>15$ years. The mode of delivery of most interventions was face to face $(74 \%)$. The intervention setting was most commonly school based (54\%), with $21 \%$ based at home. Intervention duration was less than one month in $49 \%$ of studies. Qualitative data to describe each intervention was collected using a framework based on the TIDieR checklist. We are mapping this onto behavioural frameworks (COM-B, theoretical domains framework), to categorise interventions. Our initial analysis, from 9911 participants (4719, intervention, 5192 control) from 33 studies, suggests that mean differences in interventions increase sleep duration by 11 minutes $(95 \%$ CI 6, 17 mins). However, caution is needed in interpreting this overall effect size (pooled using random effects) given the considerable heterogeneity across studies $\left(\mathrm{I}^{2}=91 \%\right)$. The influence of study design characteristics in explaining heterogeneity (including age of participants, duration and type of intervention) will be outlined.

Conclusion This review furthers our understanding of the most effective interventions to improve sleep duration in children and provides a platform to develop population level health interventions to improve sleep. Given challenges assimilating data, an overarching aim is to develop approaches that could be used to homogenise reporting in future RCTs in order that evidence can be summarised more easily.

\section{OP18 MENTAL HEALTH DISORDERS AMONGST CHILDREN AND YOUNG PEOPLE INVOLVED IN FAMILY JUSTICE COURT PROCEEDINGS: A LONGITUDINAL NATIONAL DATA LINKAGE STUDY}

'Lucy J Griffiths*, 'Joanna McGregor, ${ }^{1}$ Theodora Pouliou, ${ }^{1}$ Rhodri Johnson, ${ }^{2}$ Karen Broadhurst, ${ }^{2}$ Linda Cusworth, ${ }^{1}$ Ann John. 'Population Data Science, Swansea University Medical School, Swansea, UK; ${ }^{2}$ Centre for Child and Family Justice Research, Lancaster University, Lancaster, UK

10.1136/jech-2021-SSMabstracts. 18

Background Little is known about common mental health problems of children and young people (CYP) involved with 
the family justice system. To address this evidence gap, this study examined records of depression and anxiety in CYP involved in public and private law proceedings using linked, routinely collected, population-level-data across Wales.

Methods Retrospective e-cohort study. We calculated incidence of primary care-recorded diagnoses and symptoms of depression and anxiety of those involved in private and public law proceedings, and in a comparison group, using poisson regression. Depression and anxiety outcomes following court proceedings were evaluated using pairwise cox regression, with age and gender matched controls of CYP who had no involvement with the courts.

Results CYP in the public group had more than twice the risk of depression (adjusted Incidence Rate Ratios (aIRR): 2.2 (95\% Confidence Intervals (CI) 1.9-2.6)) and a 20\% increased risk of anxiety (aIRR: $1.2(1.0-1.5)$ ) relative to the comparison group. Those in the private group had $60 \%$ higher risk of depression (aIRR: $1.6(1.4-1.7)$ ) and a 30\% increased risk of anxiety (aIRR: $1.3(1.2-1.4)$ relative to the comparison group. Following involvement in private law proceedings, CYP were significantly more likely to have depression (Hazard Ratio (HR): 1.9 (1.7-2.1)), and anxiety (HR: 1.4 (1.2-1.5)), than the control group. Those involved in public law proceedings, were more likely to have depression than the control group (HR: 2.1 (1.7-2.6)).

Conclusion Our findings highlight the vulnerability of CYP involved in family court proceedings and increased risk of depression and anxiety. Schools, health professionals, social workers and family support workers have a key role to play in identifying mental health needs and ensuring CYP receive appropriate support both during and after proceedings.

\section{Wednesday 15 September \\ Obesity Diabetes \& Global Health, 13.00 - 15.25}

\section{OP19 PREDICTING THE RISK OF CHILDHOOD OVERWEIGHT AND OBESITY AT 10-11 YEARS USING HEALTHCARE DATA FROM PREGNANCY AND EARLY LIFE*}

${ }^{1}$ Nida Ziauddeen*, ${ }^{1}$ Paul J Roderick, ${ }^{1,2,3}$ Nisreen A Alwan. ${ }^{1}$ School of Primary Care, Population Sciences and Medical Education, Faculty of Medicine, University of Southampton, Southampton, UK; ${ }^{2}$ NIHR Southampton Biomedical Research Centre, University of Southampton and University Hospital Southampton NHS Foundation Trust, Southampton, UK; ${ }^{3}$ NIHR Applied Research Collaboration Wessex, Southampton, UK

\subsection{6/jech-2021-SSMabstracts. 19}

Background In England, 1 in 3 children aged 10-11 years live with overweight or obesity, with the prevalence in the most deprived areas being more than twice as that in the least deprived. It is important to identify children at risk of becoming overweight or obese in the future to apply early prevention interventions. We aimed to develop and internally validate prediction models of childhood overweight and obesity at age 10-11 years (Year 6) using weight and height measurements at age 4-5 years (Year R) as well as antenatal and birth data in Hampshire.

Methods A population-based anonymised linked cohort of maternal antenatal and delivery records for all births taking place at University Hospital Southampton, between 2003 to
2018 and child health records including information on postnatal growth, type of feeding and childhood body mass index (BMI) up to the age of 14 years. Childhood age- and sexadjusted BMI at 10-11 years was used to define the outcome of overweight and obesity ( $\geq 91$ st centile) in the models. Logistic regression models together with multivariable fractional polynomials were used to select model predictors and to identify transformations of continuous predictors that best predict the outcome. Models were developed in stages, incorporating data collected at 4-5 years and then first antenatal booking appointment, birth and early life predictors. Predictive accuracy was evaluated by assessing model discrimination and calibration.

Results Childhood BMI was available for 6566 children between $4-5$ years $(14.6 \%$ overweight/obese) and 10-11 years (26.1\% overweight/obese) with $10.8 \%$ overweight/obese at both ages. One-fifth of normal weight children at 4-5 years became overweight or obese by $10-11$ years, $30.3 \%$ of overweight children at 4-5 years were obese by 10-11 years and $68 \%$ of obese children remained obese. The area under the curve (AUC) was 0.82 for the model only incorporating BMI at 4-5 years and child gender. AUC increased to 0.84 on incorporating maternal predictors (BMI, smoking, age, educational attainment, ethnicity, parity, and employment status) as measured/reported at the booking appointment. Variables from birth and early life were not retained in the model.

Conclusion This prediction modelling can be applied at 4-5 years to identify the risk for later childhood overweight or obesity at 10-11 years, with improved prediction with the inclusion of pregnancy data. These prediction models demonstrate that routinely collected healthcare data can be used to target early preventive interventions.

\section{OP20 THE IMPACT OF THE SECULAR INCREASE IN BODY MASS INDEX ON HIP FRACTURE RISK IN THE NORWEGIAN POPULATION}

${ }^{1}$ Helena K Kjeldgaard*, ${ }^{1}$ Kristin Holvik, ${ }^{2}$ Martin O'Flaherty, ${ }^{3}$ Grethe S Tell, ${ }^{1,4}$ Haakon E Meyer. 'Department of Chronic Diseases and Ageing, Norwegian Institute of Public Health, Oslo, Norway; ${ }^{2}$ Department of Public Health and Policy, University of Liverpool, Liverpool, UK; ${ }^{3}$ Department of Global Public Health and Primary Care, University of Bergen, Bergen, Norway; ${ }^{4}$ Department of Community Medicine and Global Health, University of Oslo, Oslo, Norway

\subsection{6/jech-2021-SSMabstracts.20}

Background A low body mass index (BMI: weight $(\mathrm{kg}) /$ height $(m)^{2}$ ) is an important risk factor for hip fracture. In Norway, hip fracture rates have declined in recent decades at the same time as BMI has increased in the population; however, to what extent increased BMI has contributed to the declining hip fracture trend is unknown. We aimed to estimate the number of incident hip fractures prevented or postponed (IFPP) attributable to increased BMI using an adaptation of IMPACT coronary heart disease model methods.

Methods Hip fractures in Norway from 1999-2019 were extracted from the Norwegian hip fracture database (NORHip) and prevalences of BMI $>25$ in the Norwegian population was extrapolated from the fourth wave (1994-5) and the seventh wave (2015-16) of the Tromsø study. Hazard ratios of hip fracture according to age and sex were estimated using data from the Cohort of Norway 